\title{
Visualization of Three-Dimensional Acoustic Streaming Flow Patterns around an Inclined Triangular Obstruction using Digital In-Line Holographic Micro-Particle Tracking Velocimetry
}

\author{
Sheng Po HUNG and Wei-Hsin TIEN ${ }^{1}$ \\ ${ }^{1}$ Department of Mechanical Engineering, National Taiwan University of Science and Technology, Taipei, \\ Taiwan \\ whtien@mail.ntust.edu.tw
}

\begin{abstract}
Acoustic Streaming is a flow phenomenon with many applications in the field of microfluidics, such as micro mixing $[1,2]$ and particle manipulation[3]. With the manufacturing techniques evolves, more complicated geometries can be designed for microfluidic device and 3-D acoustic streaming patterns may occurs. In this study, 3-D trajectories of particle induced by acoustic streaming around an inclined triangular obstruction in a microchannel were visualized by a volumetric tracking method using Digital Inline Holographic Microscopy (DIHM)[4-6]. The triangular obstruction has a tip angle of $20^{\circ}$ and an inclined angle of $30^{\circ}$. The acoustic streaming is created under $12 \mathrm{kHz}$ oscillation of a piezo plate driven by $20 \mathrm{~V}$ voltage. Illuminated by a $450 \mathrm{~nm}$ continuous laser, the magnified hologram of the motion of $1.79 \mu \mathrm{m}$ tracer particles was recorded by a low-cost 10X industrial microscope with a machine vision camera of 10 fps (frames per second). Using RayleighSommerfeld back-propagation method[7], particle locations was reconstructed frame by frame and 3-D tracking of individual particles was performed afterwards. The trajectories of each particle were reconstructed to reveal the vortical structure of the acoustic streaming flow. For the current system setup, the measurable range was estimated to be $550 \times 685 \times 840 \mu^{3}$. The 3 D location reconstruction accuracy was verified with a calibration target and the location sensitivity was found to be linear throughout the measurable range. Reconstruction at different depth locations show that the dick-shaped calibration dots and the spherical polystyrene particles have different intensity profiles. The calibration dots show local minimum of intensity at the correct depth location, while polystyrene particles show local maximum of intensity instead. Resolved particle trajectories show that the acoustic streaming flows cause particles to move with 3-D spiral shaped motions near the side of the triangular obstruction, while particles away from the obstruction shows planar motions.
\end{abstract}

(a)

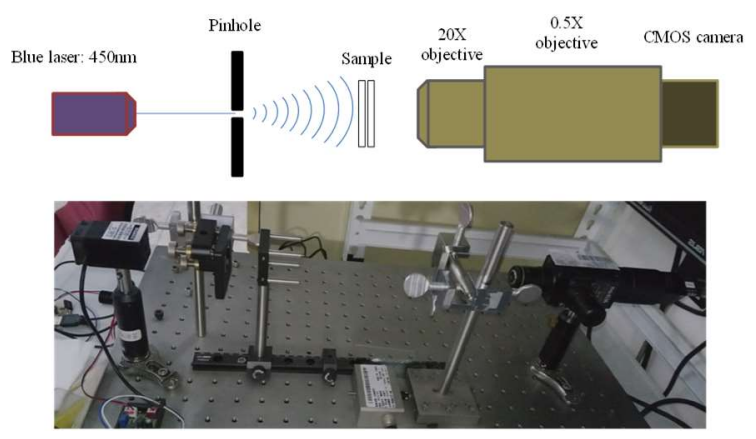

(b) Target Depth Locations Vs. Reconstructed Locations

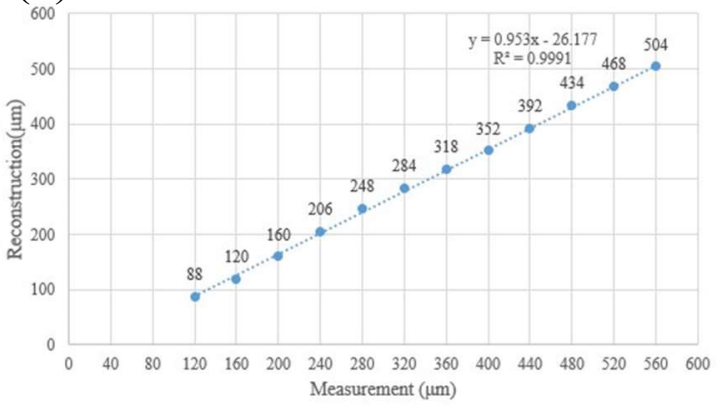

Figure 1 (a) Experimental Setup and (b) Calibration Results over the measurable range of the DIHM system. 

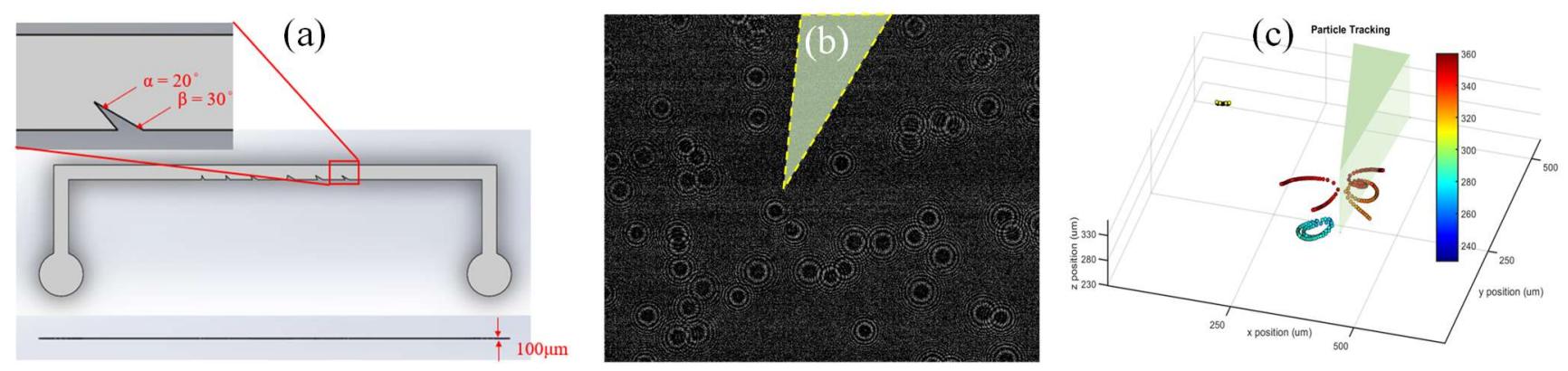

Figure 2 (a) Device Geometry (b) Magnified Hologram and (c) reconstructed particle trajectories of the acoustic streaming flow around a triangular obstruction. The obstruction has a tip angle of $20^{\circ}$ and an inclined angle of $30^{\circ}$.

\section{REFERENCES}

[1] A. Ozcelik et al., "An acoustofluidic micromixer via bubble inception and cavitation from microchannel sidewalls," (in English), Analytical Chemistry, Article vol. 86, no. 10, pp. 5083-5088, 05 / 20 / 2014.

[2] X. Mao, B. K. Juluri, M. I. Lapsley, Z. S. Stratton, and T. J. Huang, "Milliseconds microfluidic chaotic bubble mixer," (in English), Microfluidics and Nanofluidics, Article vol. 8, no. 1, pp. 139-144, 01 / 01 / 2010.

[3] M. V. Patel, I. A. Nanayakkara, M. G. Simon, and A. P. Lee, "Cavity-induced microstreaming for simultaneous onchip pumping and size-based separation of cells and particles," Lab Chip, vol. 14, no. 19, pp. 3860-72, Oct 72014.

[4] P. Memmolo et al., "Recent advances in holographic 3D particle tracking," Advances in Optics and Photonics, vol. 7, no. 4, pp. 713-755, Dec 2015.

[5] X. Yu, J. S. Hong, C. G. Liu, and M. K. Kim, "Review of digital holographic microscopy for three-dimensional profiling and tracking," Optical Engineering, vol. 53, no. 11, Nov 2014, Art. no. 112306.

[6] J. Garcia-Sucerquia, W. B. Xu, S. K. Jericho, P. Klages, M. H. Jericho, and H. J. Kreuzer, "Digital in-line holographic microscopy," (in English), Applied Optics, Article vol. 45, no. 5, pp. 836-850, Feb 2006.

[7] F. C. Cheong, B. J. Krishnatreya, and D. G. Grier, "Strategies for three-dimensional particle tracking with holographic video microscopy," Optics Express, vol. 18, no. 13, pp. 13563-13573, Jun 2010. 